\title{
Recent Trends and Changes in the Endoscopic Spinal Surgery
}

\author{
Dong Hwa Heo, ${ }^{1}$ Dong Chan Lee ${ }^{2}$, Hyeun Sung Kim³, Hungtae Chung ${ }^{1}$ \\ ${ }^{1}$ Departments of Neurosurgery, Endoscopic Spine Surgery Senter, Orthopedics, Seoul Bumin Hospital, Seoul, Republic of Korea \\ ${ }^{2}$ Department of Neurosurgery, Spine Senter, The Leon Wiltse Memorial Hospital, Anyang, Republic of Korea \\ ${ }^{3}$ Department of Neurosurgery, Nanoori Gangnam Hospital, Seoul, Spine Surgery, Seoul, Republic of Korea
}

Corresponding Author: Dong Hwa Heo, MD, $\mathrm{PhD}$ In recent years, endoscopic spine surgeries have advanced dramatically. EndosEndoscopic Spine Surgery Center, Neurosurgery, Seoul Bumin Hospital, 389, Gonghang-daero, Gangseo-gu, Seoul, Republic of Korea Tel: +82-31-240-6086

E-mail: spineneurosurgeon@gmail.com

Received: March 11, 2021

Revised: March 12, 2021

Accepted: March 14, 2021 copic spine surgeries are expanding to not only the lumbar spine, but also thoracic and cervical spines. Indications for spinal endoscopic surgery have also been expanded. Central stenosis, lateral recess stenosis, and foraminal stenosis were included in surgical indications of endoscopy. Finally, endoscopic lumbar interbody fusion surgeries have been attempted.

Key Words: Endoscopy, Spine, Biportal, Fusion

\section{INTRODUCTION}

In recent years, endoscopic spine surgeries have advanced dramatically. In addition to advancements of endoscopic spine approaches, advancements of spinal endoscopy equipment and surgical instruments have also been made. Endoscopic spine surgery was mainly used for lumbar disc herniation surgery and low back pain treatment in the past. It has now advanced to a state where laminectomy, foraminotomy, and spinal fusion are possible ${ }^{2,3,6)}$. Endoscopic spine surgeries are expanding to not only the lumbar spine, but also thoracic and cervical spines. Indications for spinal endoscopic surgery have also been greatly expanded ${ }^{8}$. Indications for endoscopic spine surgery are almost similar to those for a conventional microscopic surgery.

\section{New Systems of Endoscopic Spine Surgery}

In the past, uniportal spinal endoscopy mainly used a lumbar transforaminal approach. Spinal endoscopic surgery using a lumbar transforaminal approach was mainly performed for lumbar disc removal. Recently, interlaminar endoscopes equipped with large diameter working channel have been developed by various companies $^{7)}$. Moreover, various spinal endoscopic surgical instruments that can pass through large diameter working channel have been developed (Figure 1A). Various spinal endoscopic dissectors, water-proof drills, endoscopic Kerrison punch, and radio frequency probes can be used through the large diameter working channel of modified interlaminar uniportal endoscopic systems (Figure 1A), thus facilitating bone-work such as lamino- tomy, laminectomy, and facetectomy ${ }^{7}$. Even with a uniportal full endoscopic approach, it is possible to proceed to discectomy, decompression, and fusion through a posterior approach rather than a transforaminal approach.

Biportal endoscopic spine surgery (unilateral biportal endoscopy, UBE) introduced in 1996 was first used for lumbar discectomy ${ }^{1)}$. Compared to a uniportal endoscopic spine surgery, the surgical method of biportal endoscopy was not widely used. However, in recent years, the biportal endoscopic approach has been re-developed, mainly in South Korea. Biportal endoscopic surgery system, surgical instruments, and radiofrequency notomy, and interbody fusion using biportal endoscopy have been tried ${ }^{3,5}$. Now they are popularized. With the development of biportal and large working channel uniportal endoscopy sys-
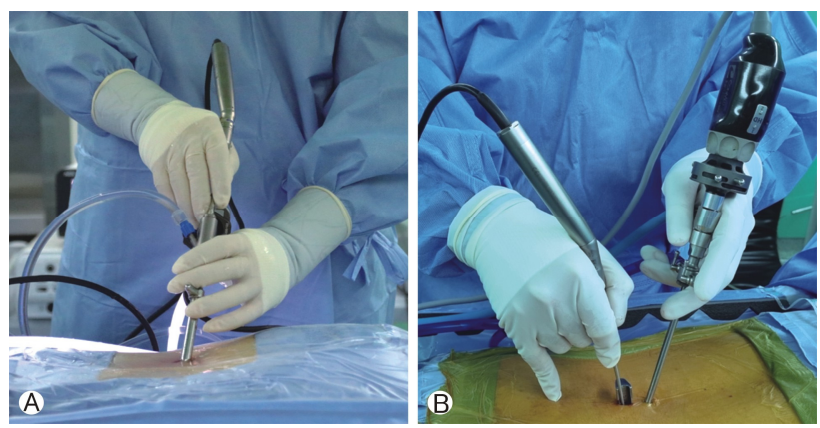

Figure 1. Recently developed endoscopy systems. (A): Modified interlaminar endoscopic system with a large working channel. (B): Biportal endoscopic systems with two channels (an endoscopic portal and a working portal). 
tems, endoscopic spine surgeries have become more advanced (Figure 1B).

\section{Paradigm shifting of Endoscopic Spine Approaches}

The development process of spinal endoscopic surgery can be largely divided into four stages (Table 1 and Figure 2). The first generation is the lumbar transforaminal approach, which has been implemented since the early days of spinal endoscopic surgery. Transforaminal endoscopic approach has been in use since its inception. The second generation is an interlaminar approach. A posterior interlaminar approach has been attempted to treat lumbosacral disc herniation, which is difficult to access with a transforaminal approach. With the third generation, surgical indication and site have developed dramatically ${ }^{6}$. With the first generation and second generation, the indication for spinal endoscopic surgery was mainly lumbar disc herniation (Figure 2 and Table 1). However, with the development of the third generation, central stenosis, lateral recess stenosis, and foraminal stenosis were included in surgical indications of endoscopy. In addition, endoscopic treatment was possible not only in the lumbar spine, but also in the cervical thoracic spine (Figure 2 and Table 1). The biggest change is shown in the fourth generation, with which endoscopic lumbar interbody fusion can be attempted. Using a posterolateral approach and a trans-kambin approach, endoscopic transforaminal lumbar interbody fusion can be performed $^{4)}$.

\section{Future of Endoscopic Spine Surgery}

Endoscopic spine surgeries including biportal and uniportal endoscopic systems are expected to continue to progress. Although endoscopic spine surgery will develop on its own, it can be combined with hybrid operation, three-dimensional surgery, navigation, and merged reality in conjunction with various other techniques. Additionally, endoscopic spine surgery might be incorporated into robotics. Endoscopic spine surgeries are expected to continue to develop with the advancement of optical techno-

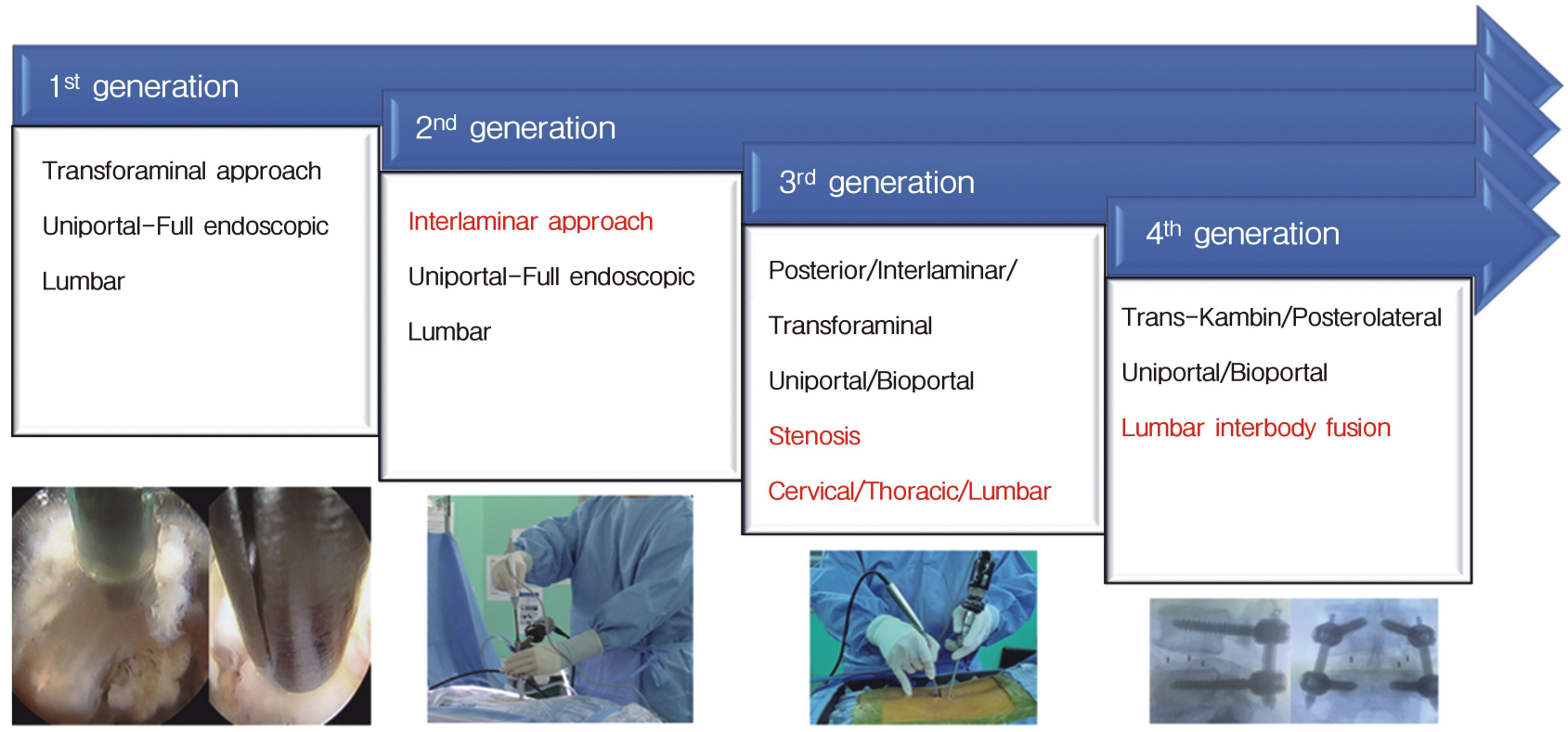

Figure 2. Generation and paradigm shifting of endoscopic spine surgeries.

Table 1. Generation and development of endoscopic spine surgeries

\begin{tabular}{|c|c|c|c|c|}
\hline & $1^{\text {st }}$ generation & $2^{\text {nd }}$ generation & $3^{\text {rd }}$ generation & $4^{\text {th }}$ generation \\
\hline Endoscopy systems & Uniportal & Uniportal & Uniportal/Biportal & Uniportal/Biportal \\
\hline Indication & Disc herniation & Disc herniation & $\begin{array}{l}\text { Disc herniation } \\
\text { Central stenosis } \\
\text { Foraminal stenosis }\end{array}$ & $\begin{array}{l}\text { Stenosis } \\
\text { Instability }\end{array}$ \\
\hline Lesion & Lumbar & Lumbar & $\begin{array}{l}\text { Lumbar } \\
\text { Thoracic } \\
\text { Cervical }\end{array}$ & $\begin{array}{l}\text { Lumbar } \\
\text { Lumbar interbody fusion }\end{array}$ \\
\hline Approach & Transforaminal & interlaminar & $\begin{array}{l}\text { Posterior } \\
\text { Interlaminar } \\
\text { Transforaminal }\end{array}$ & $\begin{array}{l}\text { Trans-Kambin } \\
\text { Posterolateral }\end{array}$ \\
\hline
\end{tabular}


logy and surgical instruments. It is believed that it will replace many parts of the conventional spine surgery.

\section{CONFLICT OF INTEREST}

Dr. $\mathrm{DH}$ Heo is an editorial board member of the journal but was not involved in the peer reviewer selection, evaluation, or decision process of this article. There are no other potential conflicts of interest relevant to this article to declare.

\section{REFERENCES}

1. De Antoni DJ, Claro ML, Poehling GG, Hughes SS: Translaminar lumbar epidural endoscopy: Anatomy, technique, and indications. Arthroscopy 12:330-334, 1996

2. Heo DH, Eum JH, Jo JY, Chung H: Modified far lateral endoscopic transforaminal lumbar interbody fusion using a biportal endoscopic approach: technical report and preliminary results. Acta Neurochir (Wien), 2021

3. Heo DH, Hong YH, Lee DC, Chung HJ, Park CK: Technique of
Biportal Endoscopic Transforaminal Lumbar Interbody Fusion. Neurospine 17:S129-S137, 2020

4. Heo DH, Lee DC, Kim HS, Park CK, Chung H: Clinical Results and Complications of Endoscopic Lumbar Interbody Fusion for Lumbar Degenerative Disease: A Meta-Analysis. World Neurosurg 145:396-404, 2021

5. Heo DH, Quillo-Olvera J, Park CK: Can Percutaneous Biportal Endoscopic Surgery Achieve Enough Canal Decompression for Degenerative Lumbar Stenosis? Prospective Case-Control Study. World Neurosurg 120:e684-e689, 2018

6. Kim M, Kim HS, Oh SW, Adsul NM, Singh R, Kashlan ON, et al.: Evolution of Spinal Endoscopic Surgery. Neurospine 16: 6-14, 2019

7. Lim KT, Meceda EJA, Park CK: Inside-Out Approach of Lumbar Endoscopic Unilateral Laminotomy for Bilateral Decompression: A Detailed Technical Description, Rationale and Outcomes. Neurospine 17:S88-S98, 2020

8. Wu PH, Kim HS, Jang IT: A Narrative Review of Development of Full-Endoscopic Lumbar Spine Surgery. Neurospine 17:S20S33, 2020 